\title{
Sobre a soma dos ângulos planos de ângulos poliédricos convexos
}

\author{
Roberto Ribeiro Paterlini(D)
}

\begin{abstract}
Resumo
Uma propriedade muito usada na teoria dos poliedros convexos é que a soma das medidas dos ângulos contíguos a um vértice deve ser estritamente menor do que $360^{\circ}$. Neste texto apresentamos uma demonstração para tal resultado, e tecemos comentários sobre algumas situações em que ele é aplicado.
\end{abstract}

Palavras-chave: Geometria; n-edros convexos; ângulo poliédrico convexo; cone poliédrico convexo; poliedros; soma de ângulos planos.

\section{Abstract}

A property widely used in the theory of convex polyhedra is that the sum of the measurements of the angles containing a vertex must be strictly less than $360^{\circ}$. In this text we present a demonstration of this result and comment on some situations in which it is applied.

Keywords: Geometry; convex polyhedral angle; convex polyhedral cone; polyhedra; sum of plan angles.

\section{Introdução}

Na classificação de poliedros convexos é muito utilizada a seguinte propriedade: a soma das medidas dos ângulos planos de qualquer ângulo poliédrico convexo é $<360^{\circ}$. Esse resultado é considerado óbvio e é aplicado sem justificativa formal nos livros didáticos atuais. Encontramos demonstrações dessa propriedade em livros didáticos antigos, como em [1], em geral usando uma definição de ângulo poliédrico não muito conveniente para aplicações em poliedros convexos.

Penso ser correto que os livros atuais não apresentem essa demonstração, por ser propriedade muito intuitiva, e, dessa forma, forma os autores não precisam sobrecarregar os livros didáticos com detalhes técnicos excessivos. É entretanto bom termos uma redação atualizada para ela.

Para exemplificar e tornar mais claras as ideias e os conceitos dos quais estamos tratando, consideremos um vértice do icosaedro truncado com faces regulares. Vemos que a ele concorrem dois hexágonos e um pentágono regulares, como está ilustrado na Figura 1. Lembrando que o ângulo de um hexágono regular mede $120^{\circ}$ e o de um pentágono regular, $108^{\circ}$, a soma dos ângulos planos ao redor desse vértice é $2 \times 120^{\circ}+108^{\circ}=348^{\circ}$, portanto $<360^{\circ}$. 
Na Figura 2 vemos que dois hexágonos e um heptágono regulares não formam um vértice de poliedro. Para isso seria necessário que as três arestas que concorrem ao vértice formassem um triedro. Mas as medidas dos ângulos planos de todo triedro devem ser $<360^{\circ}$, conforme veremos. Nesse caso temos a soma $\approx 2 \times 120^{\circ}+128,57^{\circ}=368,57^{\circ}$, que ultrapassa $360^{\circ}$.

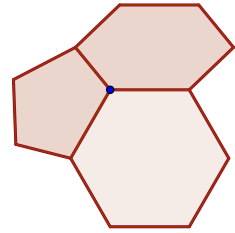

Figura 1: Triedro determinado por lados de dois hexágonos e um pentágono regulares.

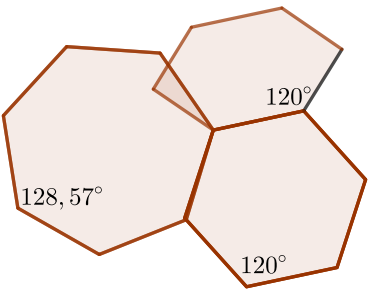

Figura 2: Dois hexágonos e um heptágono regulares não determinam um triedro.

\section{Algumas definições}

Começamos com a definição de n-edro e, em particular, de n-edro convexo. Observamos, antes, que, dados dois pontos $\mathrm{A}$ e $\mathrm{B}$, a semirreta de origem $\mathrm{A}$ e contendo $\mathrm{B}$ será anotada por $\overrightarrow{\mathrm{AB}}$. $\mathrm{O}$ leitor já sabe que se $\mathrm{C} \in \overrightarrow{\mathrm{AB}}$, com $\mathrm{C} \neq \mathrm{A}$, então $\overrightarrow{\mathrm{AC}}=\overrightarrow{\mathrm{AB}}$. Ainda, um plano $\alpha$ reparte o espaço em três conjuntos disjuntos dois a dois $\alpha, \alpha_{+}$e $\alpha_{-}$, sendo que esses dois últimos são chamados lados determinados por $\alpha$. Usaremos a notação $\angle \mathrm{ABC}$ tanto para indicar o ângulo como sua medida, e $\mathrm{AB}$ tanto para indicar o segmento quanto sua medida.

Sejam $\overrightarrow{\mathrm{OA}_{1}}, \overrightarrow{\mathrm{OA}_{2}}, \ldots, \overrightarrow{\mathrm{OA}_{\mathrm{n}}}$, com $\mathrm{n} \geq 3$, n semirretas (no espaço) com um vértice comum $\mathrm{O}$, consideradas nessa ordem (ou em reordenações circulares ou inversas), satisfazendo às seguintes condições: (i) os ângulos $\angle \mathrm{A}_{1} \mathrm{OA}_{2}, \angle \mathrm{A}_{2} \mathrm{OA}_{3}, \ldots, \angle \mathrm{A}_{\mathrm{n}-1} \mathrm{OA}_{\mathrm{n}}$ e $\angle \mathrm{A}_{\mathrm{n}} \mathrm{OA}_{1}$ têm medidas $<180^{\circ}$; (ii) os interiores desses ângulos são disjuntos dois a dois; (iii) dois ângulos contíguos quaisquer não são coplanares.

A união dessas semirretas chama-se n-edro. $\mathrm{O}$ ponto $\mathrm{O}$ é o seu vértice e as semirretas são as suas arestas. Os ângulos $\angle \mathrm{A}_{\mathrm{i}} \mathrm{OA}_{\mathrm{i}+1}(1 \leq \mathrm{i} \leq \mathrm{n}-1)$ e $\angle \mathrm{A}_{\mathrm{n}} \mathrm{OA}_{1}$, juntamente com os seus interiores, são as faces ou os ângulos planos do n-edro. O n-edro será anotado por $\hat{\mathrm{OA}} \mathrm{A}_{1} \mathrm{~A}_{2} \ldots \mathrm{A}_{\mathrm{n}}$.

Na Figura 3 vemos o desenho de um n-edro. Um caso particular é n = 3, chamado triedro, ilustrado na Figura 4. 


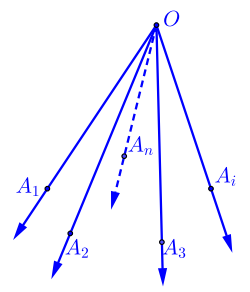

Figura 3: Desenho de um n-edro.

Vejamos agora a definição de n-edro convexo.

O n-edro $\hat{\mathrm{O}} \mathrm{A}_{1} \mathrm{~A}_{2} \ldots \mathrm{A}_{\mathrm{n}}$ será dito ser convexo quando satisfizer à seguinte condição: o plano suporte de cada face deixa o restante do n-edro contido em um de seus lados. A Figura 5 ilustra essa ideia. Por outro lado, na Figura 9 podemos ver um 6-edro não convexo.

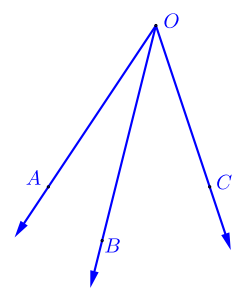

Figura 4: Um triedro.

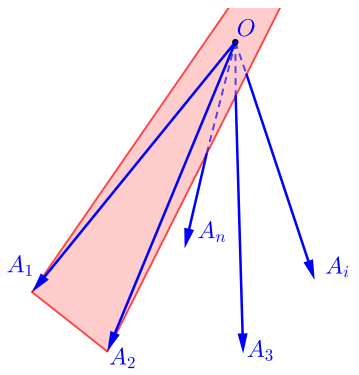

Figura 5: Ilustração de n-edro convexo.

Na literatura os n-edros são também chamados pelos nomes de ângulo poliédrico, cone poliédrico ou espaço piramidal. O nome "ângulo poliédrico" lembra-nos de observar que se considerarmos a definição usual de poliedro, como a que está apresentada em [5], seção 19.5, um vértice qualquer, juntamente com as arestas que a ele concorrem, determinam um n-edro. Se o poliedro for convexo, o n-edro também é.

\section{Uma propriedade básica dos triedros}

Notemos inicialmente que todo triedro ÔABC é convexo. Se $\alpha$ é o plano determinado por $\angle \mathrm{AOB}$, então, pela condição (iii) da definição de n-edro, $\mathrm{C} \notin \alpha$. Assim a semirreta aberta $\overrightarrow{\mathrm{OC}}-\{\mathrm{O}\}$ está contida em um dos lados de $\alpha$, e esse é todo o restante do triedro. O mesmo se aplica aos outros ângulos $\angle \mathrm{BOC}$ e $\angle \mathrm{AOC}$ : seus planos suportes deixam o restante do triedro em um só lado. Isso implica também que $\mathrm{O}$ não pertence ao plano que contém o triângulo ABC.

Propriedade 1. Em todo triedro, a medida de um ângulo plano qualquer é menor do que a soma das medidas dos outros dois.

Demonstração. Seja ÔABC um triedro. Vamos demonstrar que $\angle \mathrm{AOC}<\angle \mathrm{AOB}+\angle \mathrm{BOC}$, e basta considerar o caso em que $\angle \mathrm{AOC}>\angle \mathrm{AOB}$ e $\angle \mathrm{AOC}>\angle \mathrm{BOC}$. 
Seja $\mathrm{D}$ um ponto de $\mathrm{AC}$ tal que $\angle \mathrm{AOD}=\angle \mathrm{AOB}$. Sem perda de generalidade podemos assumir que $\mathrm{OB}=\mathrm{OD}$ (movendo o ponto $\mathrm{B}$ em sua semirreta, se necessário, o que não modifica $\angle \mathrm{AOB}$ e $\angle \mathrm{BOC}$ ). Confira a Figura 6 . Portanto $\mathrm{AOB} \cong \mathrm{AOD}$ pelo caso de congruência lado-ângulo-lado. Disso segue $\mathrm{AB}=\mathrm{AD}$. Mas no triângulo $\mathrm{ABC}$ temos $\mathrm{AC}<\mathrm{AB}+\mathrm{BC} \Rightarrow \mathrm{BC}>\mathrm{AC}-\mathrm{AB} \Rightarrow$ $\mathrm{BC}>\mathrm{AC}-\mathrm{AD} \Rightarrow \mathrm{BC}>\mathrm{DC}$.

Comparamos agora os triângulos BOC e DOC. Eles têm dois pares de lados congruentes. Em virtude do resultado conhecido com recíproca do Teorema da Dobradiça ([5], seção 9.15) dentre os ângulos por eles determinados o maior é o que se opõe ao maior lado. Como $\mathrm{DC}<\mathrm{BC}$ temos $\angle \mathrm{DOC}<\angle \mathrm{BOC}$. Portanto

$$
\begin{gathered}
\angle \mathrm{AOD}=\angle \mathrm{AOB} \Rightarrow \angle \mathrm{AOD}+\angle \mathrm{DOC}=\angle \mathrm{AOB}+\angle \mathrm{DOC} \\
\Rightarrow \angle \mathrm{AOC}=\angle \mathrm{AOB}+\angle \mathrm{DOC}<\angle \mathrm{AOB}+\angle \mathrm{BOC}
\end{gathered}
$$

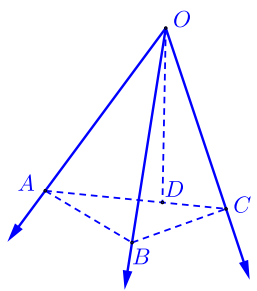

Figura 6: Ilustração da demonstração da Propriedade 1.

O mesmo vale para os outros ângulos planos: a medida de um ângulo plano qualquer do triedro é menor do que a soma das medidas dos outros dois.

A Propriedade 1 pode ser estendida para n-edros convexos quaisquer, usando o Princípio da Indução Matemática. Não vamos usar esse resultado, de modo que deixamos para o leitor conferir isso.

\section{Propriedades dos n-edros convexos}

Precisaremos da seguinte

Propriedade 2. Dado um n-edro convexo, existe um plano que contém seu vértice e que deixa todas as suas arestas em um único de seus lados, com exceção do próprio vértice.

Demonstração. Lembramos que em uma esfera $\mathrm{S}$ de centro $\mathrm{O}$, um círculo máximo é a interseção da esfera com um plano que contém O. Dois círculos máximos dizem-se perpendiculares quando seus planos suportes são perpendiculares. Dados dois pontos não antípodas de $\mathrm{S}$, existe um único círculo máximo que os contém. O arco menor desse círculo ligando os pontos chama-se geodésica com extremos nesses pontos, e o comprimento desse arco é a distância entre eles (em S). A distância de um ponto de $\mathrm{S}$ a um círculo máximo é o comprimento da geodésica determinada pelo arco menor do círculo máximo, perpendicular ao círculo dado, que passa por esse ponto e o liga a ele. Para mais detalhes confira as seções 17.6 e 21.2 de [5]. 
Tendo isso em mente, seja $\hat{O}_{1} A_{2} \ldots A_{n}$ um n-edro convexo. Consideremos uma esfera $\mathrm{S}$ com centro em O. Podemos supor, sem perda de generalidade, que os pontos $\mathrm{A}_{\mathrm{i}}, 1 \leq \mathrm{i} \leq \mathrm{n}$, estão em $\mathrm{S}$. Seja $\alpha$ o plano determinado por $\angle \mathrm{A}_{1} \mathrm{OA}_{2}$, e seja $\mathrm{C}_{1}$ o círculo máximo determinado por esse plano em $\mathrm{S}$. $\mathrm{C}_{1}$ reparte $\mathrm{S}$ em dois hemisférios abertos $\mathrm{C}_{1+}$ e $\mathrm{C}_{1-}$. Devido à convexidade do n-edro, os pontos $\mathrm{A}_{\mathrm{j}}, 3 \leq \mathrm{j} \leq \mathrm{n}$, estão em um deles, digamos que seja em $\mathrm{C}_{1+}$. Confira a Figura 7 .

Como $\angle \mathrm{A}_{1} \mathrm{OA}_{2}<180^{\circ}$ podemos escolher um diâmetro $\mathrm{AB}$ de $\mathrm{C}_{1}$ de modo que $\mathrm{A}_{1}$ e $\mathrm{A}_{2}$ estejam na mesma semicircunferência aberta determinada por esse diâmetro. Seja $\mathrm{C}_{2}$ o círculo máximo que contém $\{\mathrm{A}, \mathrm{B}\}$ e é perpendicular a $\mathrm{C}_{1}$. Também $\mathrm{C}_{2}$ reparte $\mathrm{S}$ em dois hemisférios abertos $\mathrm{C}_{2+} \mathrm{e}$ $\mathrm{C}_{2-}$, e suponhamos que $\mathrm{A}_{1}$ e $\mathrm{A}_{2}$ estejam em $\mathrm{C}_{2+}$.

Dado um ponto $\mathrm{A}_{\mathrm{j}}$ em $\mathrm{C}_{1+} \cap \mathrm{C}_{2-}$, seja $\mathrm{g}_{\mathrm{j}}$ a geodésica que o liga perpendicularmente a $\mathrm{C}_{1}$.

Seja agora $\mathrm{CD}$ o diâmetro de $\mathrm{C}_{1}$ perpendicular a $\mathrm{AB}$, com $\mathrm{C} \in \mathrm{C}_{2+}$. Seja $\mathrm{C}_{3}$ o círculo máximo que contém $\{\mathrm{C}, \mathrm{D}\}$ e é perpendicular a $\mathrm{C}_{1}$. Tomemos em $\mathrm{C}_{3}$ um ponto E situado em $\mathrm{C}_{1-} \cap \mathrm{C}_{2+}$ de forma que a distância de $\mathrm{C}$ a $\mathrm{E}$ seja menor do que a medida de qualquer $\mathrm{g}_{\mathrm{j}}$. Seja $\mathrm{C}_{4}$ o círculo máximo que contém $\{\mathrm{A}, \mathrm{B}, \mathrm{E}\}$. Esse círculo reparte $\mathrm{S}$ em dois hemisférios abertos, e suponhamos que $A_{1}$ e $A_{2}$ estejam em $C_{4+}$. Todos os pontos $A_{i}$ de $C_{1+} \cap C_{2+}$ e de $C_{2}$ também estão em $C_{4+}$. Vemos que $C_{4}$ intersecta as geodésicas $g_{j}$ fora de $A_{j}$, portanto esses pontos $A_{j}$ também estão em $\mathrm{C}_{4+}$.

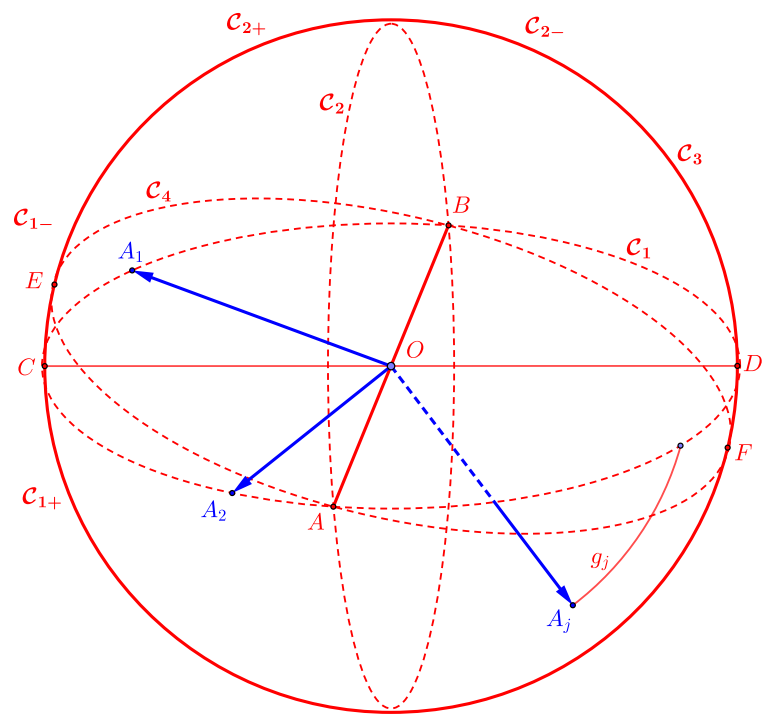

Figura 7: Ilustração da demonstração da Propriedade 2.

Finalmente, seja $\beta$ o plano determinado por $\mathrm{C}_{4}$. Vemos que $\beta$ contém $\mathrm{O}$. Ainda, os pontos $\mathrm{A}_{\mathrm{i}}$, $1 \leq \mathrm{i} \leq \mathrm{n}$, estão do mesmo lado desse plano. Assim todas as semirretas $\overrightarrow{\mathrm{OA}_{\mathrm{i}}}, 1 \leq \mathrm{i} \leq \mathrm{n}$, estão do mesmo lado de $\beta$, com exceção de $\mathrm{O}$.

Vejamos agora a

Propriedade 3. Dado um n-edro convexo, existe um plano que não contém seu vértice e que intersecta todas as suas arestas. Ainda mais, se o $\mathrm{n}$-edro é $\hat{\mathrm{O}}_{1} \mathrm{~A}_{2} \ldots \mathrm{A}_{\mathrm{n}}$, e indicando por $\mathrm{B}_{\mathrm{i}}$ a interseção de $\overrightarrow{\mathrm{OA}_{\mathrm{i}}}$ com o plano referido, então $\mathrm{B}_{1} \mathrm{~B}_{2} \ldots \mathrm{B}_{\mathrm{n}}$ é um polígono convexo. 
Demonstração. Devido à Propriedade 2 existe um plano $\alpha$ que contém $\mathrm{O}$ e que deixa as arestas do n-edro do mesmo lado, com exceção do vértice. Consideremos um plano $\beta$ paralelo a $\alpha$ e situado do mesmo lado de $\alpha$ em que está o restante do n-edro. Nenhuma das arestas do n-edro é paralela a $\beta$ (caso contrário seria paralela a $\alpha$, e como $\mathrm{O} \in \alpha$ a aresta estaria contida em $\alpha$, gerando uma contradição). Consequentemente a reta $\overleftrightarrow{\mathrm{OA}_{\mathrm{i}}}$ encontra $\beta$, e esse encontro tem que ser em $\overrightarrow{\mathrm{OA}_{\mathrm{i}}}$, pois é a parte da reta que está do mesmo lado de $\alpha$ que $\beta$. Assim existem os pontos $\mathrm{B}_{\mathrm{i}}=\beta \cap \overrightarrow{\mathrm{OA}_{\mathrm{i}}}$, $1 \leq \mathrm{i} \leq \mathrm{n}$.

Observemos que $\mathrm{B}_{1} \mathrm{~B}_{2} \ldots \mathrm{B}_{\mathrm{n}}$ é um polígono. De fato, os pontos $\mathrm{B}_{\mathrm{i}}$ são diferentes dois a dois, pois as arestas do n-edro também são. Dois lados contíguos da linha poligonal fechada $\mathrm{B}_{1} \mathrm{~B}_{2} \ldots \mathrm{B}_{\mathrm{n}}$ não podem ser colineares, caso contrário as faces do n-edro que os contêm seriam coplanares, o que contraria a condição (iii) da definição de n-edro. Devido à condição (ii) da mesma definição, os lados da linha poligonal não se intersectam a não ser nas suas extremidades. Portanto $B_{1} B_{2} \ldots B_{n}$ é um polígono.

Aplicando a definição de convexidade de n-edro, vemos que a reta suporte de qualquer aresta de $\mathrm{B}_{1} \mathrm{~B}_{2} \ldots \mathrm{B}_{\mathrm{n}}$ deixa o restante do polígono em um único lado relativamente ao plano $\beta$. Dessa forma, $\mathrm{B}_{1} \mathrm{~B}_{2} \ldots \mathrm{B}_{\mathrm{n}}$ é convexo.

Nota 1. Em livros didáticos antigos, por exemplo [1], os autores definem ângulo poliédrico já supondo a existência do polígono convexo $\mathrm{B}_{1} \mathrm{~B}_{2} \ldots \mathrm{B}_{\mathrm{n}}$ obtido na Propriedade 3 . Com isso os autores evitam a necessidade de demonstrar as propriedades 2 e 3. Entretanto nossa definição é mais atualizada e permite aplicar os resultados aqui obtidos aos poliedros convexos.

Finalmente temos o

Teorema 1. Em qualquer n-edro convexo, a soma das medidas dos seus ângulos planos é $<360^{\circ}$.

Demonstração. Seja $\hat{O}_{1} \mathrm{~A}_{2} \ldots \mathrm{A}_{\mathrm{n}}$ um n-edro convexo, $\mathrm{n} \geq 3$. Usando a Propriedade 3 podemos supor que os pontos $A_{i}, 1 \leq \mathrm{i} \leq \mathrm{n}$ são coplanares, e que $\mathrm{A}_{1} \mathrm{~A}_{2} \ldots \mathrm{A}_{\mathrm{n}}$ é um polígono convexo. Acompanhemos a Figura 8 .

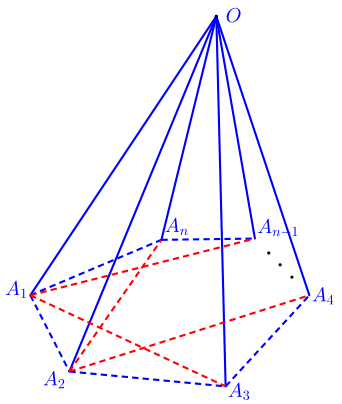

Figura 8: Ilustração da demonstração do Teorema 1.

Uma forma elegante de demonstrar a afirmação é usar repetidamente a Propriedade 1. Temos 


$$
\begin{array}{cccc}
\hat{\mathrm{A}}_{1} \mathrm{~A}_{\mathrm{n}} \mathrm{A}_{2} \mathrm{O} \text { é um triedro } & \Rightarrow & \angle \mathrm{A}_{\mathrm{n}} \mathrm{A}_{1} \mathrm{~A}_{2} & <\angle \mathrm{A}_{2} \mathrm{~A}_{1} \mathrm{O}+\angle \mathrm{A}_{\mathrm{n}} \mathrm{A}_{1} \mathrm{O} \\
\hat{\mathrm{A}}_{2} \mathrm{~A}_{1} \mathrm{~A}_{3} \mathrm{O} \text { é um triedro } & \Rightarrow & \angle \mathrm{A}_{1} \mathrm{~A}_{2} \mathrm{~A}_{3}<\angle \mathrm{A}_{1} \mathrm{~A}_{2} \mathrm{O}+\angle \mathrm{A}_{3} \mathrm{~A}_{2} \mathrm{O} \\
\hat{\mathrm{A}}_{3} \mathrm{~A}_{2} \mathrm{~A}_{4} \mathrm{O} \text { é um triedro } & \Rightarrow & \angle \mathrm{A}_{2} \mathrm{~A}_{3} \mathrm{~A}_{4}<\angle \mathrm{A}_{2} \mathrm{~A}_{3} \mathrm{O}+\angle \mathrm{A}_{4} \mathrm{~A}_{3} \mathrm{O} \\
\vdots & & \\
\hat{\mathrm{A}}_{\mathrm{n}} \mathrm{A}_{\mathrm{n}-1} \mathrm{~A}_{1} \mathrm{O} \text { é um triedro } & \Rightarrow & \angle \mathrm{A}_{\mathrm{n}-1} \mathrm{~A}_{\mathrm{n}} \mathrm{A}_{1}<\angle \mathrm{A}_{\mathrm{n}-1} \mathrm{~A}_{\mathrm{n}} \mathrm{O}+\angle \mathrm{A}_{1} \mathrm{~A}_{\mathrm{n}} \mathrm{O}
\end{array}
$$

Somando membro a membro as desigualdades, no lado esquerdo temos a soma dos ângulos do polígono $A_{1} A_{2} \ldots A_{n}$, portanto essa soma vale $(n-2) 180^{\circ}$. Do lado direito temos a soma dos ângulos dos vértices $A_{1}, A_{2}, \ldots, A_{n}$ dos triângulos $A_{1} \mathrm{OA}_{2}, A_{2} \mathrm{OA}_{3}, \ldots, \mathrm{A}_{\mathrm{n}} \mathrm{OA}_{1}$. Logo essa soma vale $n 180^{\circ}-\mathrm{S}$, em que $\mathrm{S}$ é a soma dos ângulos planos do n-edro.

Portanto

$$
(\mathrm{n}-2) 180^{\circ}<\mathrm{n} 180^{\circ}-\mathrm{S} \Rightarrow \mathrm{S}<360^{\circ}
$$

e terminamos a demonstração.

Notemos que o esquema da demonstração do Teorema 1 funciona perfeitamente para $\mathrm{n}=3$.

\section{Um contraexemplo para o caso não convexo}

Se o poliedro não for convexo então a soma dos ângulos de alguns de seus n-edros pode ser $>360^{\circ}$. Vemos um exemplo na Figura 9, em que o poliedro é formado pela junção de seis cubos. Esse poliedro é claramente não convexo. $\mathrm{O}$ vértice destacado, juntamente com as arestas que a ele concorrem, define um 6-edro cujos ângulos planos medem $90^{\circ}$. Portanto a soma dos ângulos planos desse 6 -edro é $6 \times 90^{\circ}=540^{\circ}$.

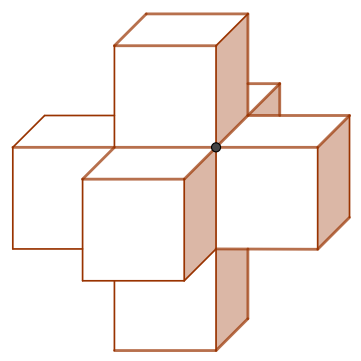

Figura 9: Poliedro não convexo formado pela junção de seis cubos.

Nota 2. Aproveitamos para observar que $360^{\circ}$ é o melhor valor possível para o Teorema 1 (isto é, não podemos colocar no lugar de $360^{\circ}$ um valor menor). Para constatar isso consideremos os prismas com todas as faces regulares (um exemplo está desenhado na Figura 11). Em um vértice qualquer concorrem dois quadrados e um polígono regular de lado $n \geq 3$. Portanto a soma dos ângulos planos em redor de um vértice é $\mathrm{S}=90^{\circ}+90^{\circ}+\alpha_{\mathrm{n}}=180^{\circ}+\alpha_{\mathrm{n}}$, em que $\alpha_{\mathrm{n}}$ é o ângulo do polígono. Mas $\alpha_{\mathrm{n}}=(\mathrm{n}-2) 180^{\circ} / \mathrm{n}=180^{\circ}-\frac{2}{\mathrm{n}} 180^{\circ}$ pode ser tão próximo de $180^{\circ}$ quanto quisermos para $\mathrm{n}$ suficientemente grande, e assim $\mathrm{S}$ é menor do que $360^{\circ}$ e é tão próximo desse valor quanto quisermos.

\section{Aplicações à classificação de poliedros convexos com faces regulares}

O uso do Teorema 1 pode facilitar a classificação da família dos poliedros de Platão. São poliedros convexos formados por faces regulares de um só tipo e com todos os vértices de um único tipo (aos quais concorrem sempre a mesma quantidade de arestas). 
Observemos que a um vértice de um poliedro qualquer sempre concorrem pelo menos três faces. Em um poliedro de Platão essas faces não podem ser hexágonos regulares, pois seus ângulos medem $120^{\circ}$ e 3 deles perfazem $360^{\circ}$, o que contraria o Teorema 1 . Polígonos com 7 lados ou mais têm ângulos ainda maiores, de modo que um poliedro de Platão só pode ser formado por triângulos equiláteros, quadrados ou pentágonos regulares.

Dado um poliedro de Platão, seja n a quantidade de lados de cada face, e seja p a quantidade de arestas que concorrem a cada vértice. Cada vértice forma um ângulo poliédrico convexo, ou um $\mathrm{p}$-edro, de modo que $\mathrm{p} \geq 3$. Já vimos que $\mathrm{n}=3$, ou $\mathrm{n}=4$ ou $\mathrm{n}=5$. Vejamos o que ocorre em cada caso. Observemos que, dado um poliedro de Platão, se F é a quantidade de faces, A a de arestas e $\mathrm{V}$ a de vértices, então a contagem desses elementos fornece-nos duas relações:

$$
2 \mathrm{~A}=\mathrm{nF} \quad \mathrm{e} \quad \mathrm{pV}=\mathrm{nF}
$$

Lembremos também a relação de Euler:

$$
\mathrm{V}-\mathrm{A}+\mathrm{F}=2
$$

Caso 1: $\mathrm{n}=5$. Como as faces são pentágonos regulares e seu ângulo mede $108^{\circ}$, a quantidade de faces que concorre em cada vértice não pode ultrapassar 3, devido ao Teorema 1. Portanto $\mathrm{n}=5$ implica $\mathrm{p}=3$. As relações $(1)$ tornam-se $2 \mathrm{~A}=5 \mathrm{~F}$ e $3 \mathrm{~V}=5 \mathrm{~F}$. De $\mathrm{V}-\mathrm{A}+\mathrm{F}=2$ temos $6 \mathrm{~V}-6 \mathrm{~A}+6 \mathrm{~F}=12 \Rightarrow 10 \mathrm{~F}-15 \mathrm{~F}+6 \mathrm{~F}=12 \Rightarrow \mathrm{F}=12$. Voltando às duas primeiras relações vem $2 \mathrm{~A}=5 \times 12=60 \Rightarrow \mathrm{A}=30$ e $3 \mathrm{~V}=60 \Rightarrow \mathrm{V}=20$. O dodecaedro regular tem esses valores (Figura 10, desenho da direita).

Caso 2: $\mathrm{n}=4$. Como as faces são quadrados e seus ângulos medem $90^{\circ}$, a quantidade de faces que concorre em cada vértice não pode ultrapassar 3, novamente devido ao Teorema 1 . Portanto $\mathrm{n}=4$ implica $\mathrm{p}=3$. As relações (1) tornam-se $2 \mathrm{~A}=4 \mathrm{~F}$ e $3 \mathrm{~V}=4 \mathrm{~F}$. Procedendo de modo análogo ao caso 1 obtemos $\mathrm{F}=6, \mathrm{~A}=12$ e $\mathrm{V}=8$. O hexaedro regular, ou cubo, tem esses valores (Figura 10, segundo desenho da esquerda para a direita).

Caso 3: $\mathrm{n}=3$. Como as faces são triângulos equiláteros e seus ângulos medem $60^{\circ}$, o Teorema 1 diz-nos que podemos ter $\mathrm{p}=3, \mathrm{p}=4$ ou $\mathrm{p}=5$. Procedendo de modo análogo ao caso 1 temos:

Se $n=3$ e $p=3$ obtemos $F=4, A=6$ e $V=4$, o que corresponde ao tetraedro regular.

Se $\mathrm{n}=3$ e $\mathrm{p}=4$ obtemos $\mathrm{F}=8, \mathrm{~A}=12$ e $\mathrm{V}=6$, o que corresponde ao octaedro regular.

Se $n=3$ e p $=5$ obtemos $F=20, A=30$ e V = 12, o que corresponde ao icosaedro regular.

Esses sólidos estão desenhados na Figura 10. Sua construção pode ser feita manualmente, por exemplo, usando planificações, ou através de coordenadas cartesianas ou usando outras técnicas. Dessa forma podemos constatar que existem e são únicos. Em resumo temos a

Propriedade 4. Existem apenas cinco poliedros convexos com todas as faces regulares do mesmo tipo e todos os vértices do mesmo tipo. São: o tetraedro, o hexaedro, o octaedro, o icosaedro e o dodecaedro (todos regulares).

Para estudar mais detalhes sobre os poliedros de Platão (em particular, sua construção) confira, por exemplo, [3], a partir da pág. 327. Em [6], a partir da página 26, os autores explicam como construir, com dobraduras, o cubo, o tetraedro, o octaedro e o icosaedro. 

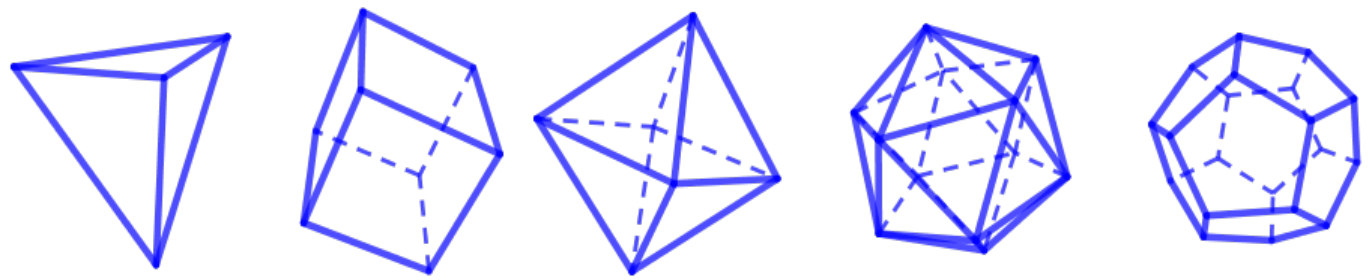

Figura 10: Os poliedros de Platão. Da esquerda para a direita: o tetraedro, o hexaedro, o octaedro, o icosaedro e o dodecaedro (todos regulares).

O Teorema 1 auxilia bastante na classificação da próxima família de poliedros convexos, os chamados sólidos de Arquimedes. São poliedros convexos semirregulares que não pertencem às famílias dos prismas e dos antiprismas. Poliedros semirregulares são aqueles que satisfazem às duas condições seguintes: (i) todas as faces são regulares mas não do mesmo tipo e (ii) todos os vértices têm a mesma configuração cíclica.

Existem 13 sólidos de Arquimedes. Uma classificação é apresentada em [6] a partir da página 57. Confira também [4], a partir da página 181.

Exemplos de prisma e antiprisma podem ser vistos nas Figuras 11 e 12.

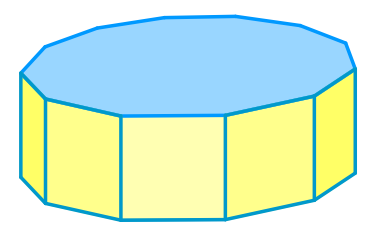

Figura 11. Prisma dodecagonal com faces regulares.

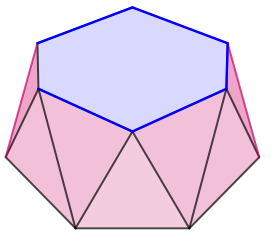

Figura 12: Antiprisma hexagonal com faces regulares.

A afirmação do Teorema 1 é usada ainda na classificação das famílias restantes de poliedros convexos cujas faces são polígonos regulares. Faremos apenas algumas observações parciais, já que se trata de um assunto bastante longo.

Já comentamos que se a um vértice de um poliedro convexo concorrem apenas triângulos equiláteros, podemos ter: (i) 3 triângulos, como no tetraedro regular (desenho da esquerda na Figura 10); (ii) 4 triângulos, como no vértice da pirâmide de base quadrada (Figura 13); (iii) 5 triângulos, como no vértice da pirâmide cuja base é um pentágono regular (Figura 14).

Levando em conta que as faces laterais de uma pirâmide são sempre triângulos, temos a seguinte conclusão:

Propriedade 5. Existem apenas três pirâmides cujas faces são regulares: a de base triangular regular (o tetraedro regular), a de base quadrada e a de base pentagonal regular.

As considerações acima nos fazem ver ainda que, em qualquer vértice de um poliedro convexo cujas faces são regulares, concorrem no máximo cinco faces. De fato, para obter o maior número de faces 


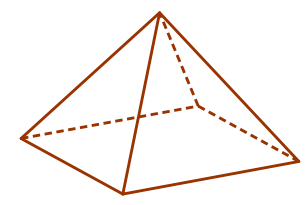

Figura 13: Pirâmide quadrada regular.

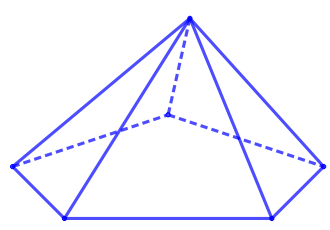

Figura 14: Pirâmide pentagonal regular.

precisamos considerar a que tem o ângulo de menor medida, que é o triângulo equilátero. Mas já vimos que o máximo é 5 .

Por outro lado, se a um vértice concorrem 5 faces, pelo menos quatro precisam ser triângulos equiláteros. De fato, se existirem apenas três, as outras duas têm ângulos $\geq 90^{\circ}$. Assim a soma dos ângulos planos no 5-edro seria

$$
\mathrm{S} \geq 60^{\circ}+60^{\circ}+60^{\circ}+90^{\circ}+90^{\circ}=360^{\circ}
$$

e já vimos que isso não é possível.

Portanto, pelo menos quatro faces são necessariamente triângulos equiláteros. Essas faces perfazem $4 \times 60^{\circ}=240^{\circ}$. Como $360^{\circ}-240^{\circ}=120^{\circ}$, a quinta face precisa ter ângulo $<120^{\circ}$. Portanto a quinta face precisa ser outro triângulo equilátero, um quadrado ou um pentágono regular (ângulo de $108^{\circ}$ ). Qualquer outro polígono regular com n $\geq 6$ lados não é possível, pois tem ângulo $\geq 120^{\circ}$.

Temos assim a

Propriedade 6. Existem apenas três tipos de vértices de poliedros convexos aos quais concorrem cinco faces regulares: (i) as cinco faces são triângulos equiláteros; (ii) quatro faces são triângulos equiláteros e a quinta é um quadrado; (iii) quatro faces são triângulos equiláteros e a quinta é um pentágono regular.

O tipo (i) comentado na Propriedade 6 ocorre, por exemplo, na pirâmide pentagonal (Figura 14) e também no icosaedro regular (quarto desenho da esquerda para a direita na Figura 10). As Figuras 15 e 16 apresentam exemplos para os outros dois tipos.

Consideremos agora um vértice do tipo 4 , ao qual concorrem as faces regulares $f_{a}, f_{b}, f_{c}$ e $f_{d}$, de tipos a, b, c e d, respectivamente, com $\mathrm{a} \leq \mathrm{b} \leq \mathrm{c} \leq \mathrm{d}$, e ângulos de medida $\alpha, \beta, \gamma$ e $\delta$, respectivamente. Sabemos que o ângulo do polígono regular de n lados é $\alpha_{\mathrm{n}}=(\mathrm{n}-2) 180^{\circ} / \mathrm{n}$. Com um cálculo simples podemos mostrar que $\mathrm{n} \rightarrow \alpha_{\mathrm{n}}$ é crescente. Portanto $\alpha \leq \beta \leq \gamma \leq \delta$.

Em virtude do Teorema 1 temos $\alpha+\beta+\gamma+\delta<360^{\circ}$, e vemos que nem todos esses valores podem ser $\geq 90^{\circ}$. Portanto $\mathrm{f}_{\mathrm{a}}$ é um triângulo regular e $\mathrm{a}=3$. Como $\alpha=60^{\circ}$ temos $\beta+\gamma+\delta<300^{\circ}$. Mas esses valores não podem ser todos $\geq 108^{\circ}$ (ângulo do pentágono regular), de modo que $\mathrm{b}=3$ ou $\mathrm{b}=4$.

Se $\mathrm{b}=4$ temos $\beta=90^{\circ}$ e $\gamma+\delta<210^{\circ}$, e esses dois valores não podem ser ambos $\geq 108^{\circ}$. Portanto $\mathrm{b}=4$ implica $\mathrm{c}=4$. Então $\gamma=90^{\circ}$ e segue $\delta<120^{\circ} \operatorname{com~} \mathrm{d}=4$ ou $\mathrm{d}=5$.

Suponhamos $\mathrm{b}=3$, com $\beta=60^{\circ}$. Temos $\gamma+\delta<240^{\circ}$, e assim c e d não podem ser ambos $\geq 6$ ( o ângulo do hexágono regular é $120^{\circ}$ ). Portanto $3 \leq \mathrm{c} \leq 5$. 


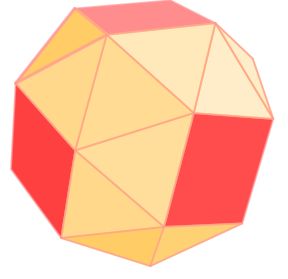

Figura 15: O cubo aumentado: em cada vértice concorrem quatro triângulos equiláteros e um quadrado.

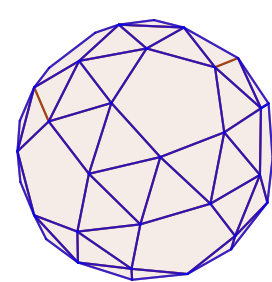

Figura 16: Dodecaedro aumentado: em cada vértice concorrem quatro triângulos equiláteros e um pentágono regular.

Se c $=3$ temos $\gamma=60^{\circ}$, e a condição sobre $\delta$ é $\delta<180^{\circ}$. Assim d pode ter qualquer valor $\mathrm{d} \geq 3$.

Se c $=4$ temos $\gamma=90^{\circ}$, e a condição sobre $\delta$ é $\delta<150^{\circ}$. Como o ângulo do dodecágono regular mede $150^{\circ}$ obtemos, $4 \leq \mathrm{d} \leq 11$.

Se c $=5$ temos $\gamma=108^{\circ}$, e a condição sobre $\delta$ é $\delta<132^{\circ}$. Como o ângulo do heptágono regular mede $\approx 128,57^{\circ}$ e o do octógono regular, $135^{\circ}$, temos $5 \leq \mathrm{d} \leq 7$.

Podemos organizar essas conclusões da seguinte forma:

Propriedade 7. Suponhamos que a um vértice concorram quatro faces regulares, formando um 4-edro convexo. Temos as seguintes possibilidades:

(i) três faces são triângulos regulares e a quarta é um polígono regular com qualquer número de lados;

(ii) duas faces são triângulos regulares, a terceira é um quadrado e a quarta é um polígono regular com $\mathrm{n}$ lados, $4 \leq \mathrm{n} \leq 11$;

(iii) duas faces são triângulos regulares, a terceira é um pentágono regular e a quarta é um polígono regular com $\mathrm{n}$ lados, $5 \leq \mathrm{n} \leq 7$;

(iv) uma face é um triângulo regular e as outras três são quadrados;

(v) uma face é um triângulo regular, outras duas são quadrados e a quarta é um pentágono regular.

Podemos examinar ainda os triedros gerados por faces regulares. Procedendo de forma análoga à que fizemos acima temos:

Propriedade 8. Suponhamos que a um vértice concorram três faces regulares, formando um triedro. Temos as seguintes possibilidades:

(i) três faces são triângulos regulares;

(ii) duas faces são triângulos regulares, e a terceira é um polígono regular com n lados, $4 \leq \mathrm{n}$;

(iii) uma face é um triângulo regular, a segunda é um quadrado, um pentágono ou um hexágono (regulares), e a terceira é um polígono regular com número de lados maior ou igual do que o da segunda face; 
(iv) uma face é um triângulo regular, a segunda é um heptágono ou um octógono ou um eneágono ou um decágono ou um undecágono (regulares), e a terceira é um polígono regular com número de lados $\mathrm{n}$ satisfazendo, respectivamente, $7 \leq \mathrm{n} \leq 41,8 \leq \mathrm{n} \leq 23,9 \leq \mathrm{n} \leq 17,10 \leq \mathrm{n} \leq 14 e$ $11 \leq \mathrm{n} \leq 13$

(v) três faces são quadrados;

(vi) duas faces são quadrados e a terceira é um polígono regular com número de lados $\mathrm{n} \geq 5$;

(vii) uma face é um quadrado, a segunda é um pentágono ou um hexágono ou um heptágono regulares, e a terceira um polígono regular com $\mathrm{n}$ lados satisfazendo, respectivamente, $5 \leq \mathrm{n} \leq 19$, $6 \leq \mathrm{n} \leq 11$ e $7 \leq \mathrm{n} \leq 9 ;$

(viii) três faces são pentágonos regulares;

(ix) duas faces são pentágonos regulares e a terceira é um polígono regular com número de lados $6 \leq \mathrm{n} \leq 9$;

(x) uma face é um pentágono regular, outra face é um hexágono regular e a terceira é um hexágono ou um heptágono regulares.

Nota 3. Observamos que alguns dos casos descritos acima já foram obtidos como vértices dos poliedros de Platão ou fazem parte dos sólidos de Arquimedes. O antiprisma hexagonal (Figura 12) exemplifica o caso (i) da Propriedade 7, e o prisma dodecagonal com faces regulares (Figura 11) exemplifica o caso (vi) da Propriedade 8.

Entretanto vários dos casos descritos nas Propriedades 7 e 8 ocorrem, a princípio, apenas como ângulos poliédricos. Muitos deles não fazem parte de poliedros convexos com faces regulares. Daremos mais detalhes adiante, na Nota 6.

Nota 4. Norman W. Johnson elaborou, em 1966, uma lista de 92 poliedros convexos com faces regulares (chamados sólidos de Johnson). Para ver a lista completa desses sólidos e as referências consulte [7]. Victor A. Zalgaller provou, também em 1966, que não existem outros.

Da lista de Johnson não fazem parte os poliedros de Platão, os sólidos de Arquimedes, os prismas e os antiprismas. Portanto os poliedros convexos com faces regulares podem ser:

(a) os cinco sólidos de Platão (poliedros regulares);

(b) os treze sólidos de Arquimedes;

(c) os prismas com faces regulares (existem infinitos);

(d) os antiprismas com faces regulares (existem infinitos);

(e) os sólidos de Johnson, em número de 92.

Sobre as três pirâmides mencionadas na Propriedade 5, o tetraedro regular é um sólido de Platão, e as outras duas são sólidos de Johnson. Os poliedros das Figuras 15 e 16 são sólidos de Arquimedes. Nas Figuras 11 e 12 já vimos exemplos de prisma e antiprisma.

Por outro lado, na Figura 17 temos o desenho do sólido de Johnson denominado "bipirâmide quadrada giralongada". Ele é formado pela junção de duas pirâmides quadradas com um antiprisma de bases quadradas. Tem 16 faces (que são triângulos equiláteros), 24 arestas e 10 vértices, sendo 2 do tipo 4 e 8 do tipo 5. A Figura 18 mostra uma das suas planificações, e usá-la é certamente a forma mais fácil para se construir um modelo desse poliedro. 


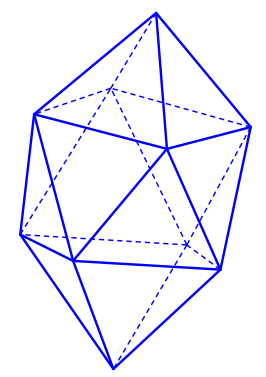

Figura 17: Bipirâmide quadrada giralongada.

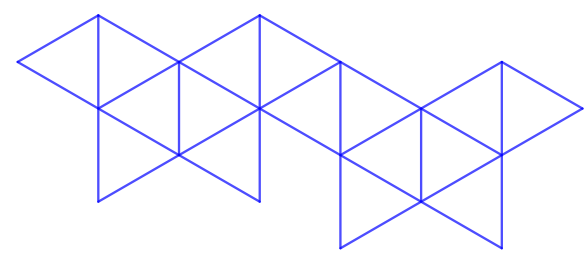

Figura 18: Uma planificação da bipirâmide quadrada giralongada.

Nota 5. Vimos que, em um poliedro convexo, a soma $\mathrm{s}_{\mathrm{i}}$ dos ângulos das faces que circundam o vértice i e que são contíguos a esse vértice satisfaz à desigualdade $\mathrm{s}_{\mathrm{i}}<360^{\circ}$. A diferença $\delta_{\mathrm{i}}=360^{\circ}-\mathrm{s}_{\mathrm{i}}$ chama-se deficiência do vértice i. Um Teorema de Descartes diz que a soma das deficiências em qualquer poliedro convexo é

$$
\delta_{1}+\delta_{2}+\ldots+\delta_{\mathrm{V}}=720^{\circ}
$$

O Teorema de Descartes é equivalente à fórmula de Euler $\mathrm{V}-\mathrm{A}+\mathrm{F}=2$. Mais detalhes podem ser lidos em [4], a partir da pág. 172.

Nota 6. Estivemos estudando na Seção 6 condições para a existência de vértices de certos tipos em poliedros convexos. É bom observarmos que são condições locais. Constatada a possibilidade de ocorrência de um vértice com determinada configuração, é necessário examinar se ele combina com outros vértices para formar um poliedro. Uma forma de fazer isso consiste em obter restrições adicionais, como o uso de fórmulas combinatórias e o exame das medidas dos diedros.

A título de informação complementar, um teorema de Grünbaum e Johnson [2] afirma que os poliedros convexos com faces regulares, e que não sejam das famílias dos prismas e dos antiprismas, são finitos e neles só podem ocorrer como faces triângulos, quadrados, pentágonos, hexágonos, octógonos e decágonos (todos regulares). Esse teorema ajuda bastante na construção dos 92 sólidos de Johnson.

\section{Referências}

[1] Fouet, E. A. Leçons de Géométrie Élémentaire. Paris: Librairie Vuibert, 1924.

[2] Grünbaum, B. e Johnson, N. W. "The Faces of a Regular-Faced Polyhedron. Journal of the London Mathematical Society,"Volume s1-40, Issue 1, 1965, Pages 577-586.

[3] Neto, A. C. M. Geometria. Rio de Janeiro: Sociedade Brasileira de Matemática, 2013.

[4] Oliveira, L. A. T. "Poliedros. "In: Sá, C. C. e Rocha, J. (Ed.) Treze Viagens pelo Mundo da Matemática. 2a ed. Rio de Janeiro: Sociedade Brasileira de Matemática, 2012. p. 161-194.

[5] Paterlini, R. R. Geometria Elementar, gênese e desenvolvimento. Disponível em <https://www. dm.ufscar.br/ ptlini/livros/livro_geo.html> Acesso em: 14 de maio de 2019. 
[6] Sampaio, J. C. V. et alii. Geometria Espacial. Coleção Matem@tica na Pr@tica. Cuiabá: Central de Texto, 2013. Para obter este texto solicite o arquivo .pdf ao autor Prof. João Carlos Vieira Sampaio, do Departamento de Matemática da UFSCar.

[7] Wikipédia. Sólidos de Johnson. Disponível em <https://pt.wikipedia.org/wiki/Solidos_de_ Johnson> Acesso em: 14 de maio de 2019.

Roberto Ribeiro Paterlini Universidade Federal de São Carlos $<$ roberto@dm.ufscar.br>

Recebido: $02 / 01 / 2020$

Publicado: $15 / 04 / 2019$ 